\title{
NILPOTENCY OF DERIVATIONS. II
}

\author{
L. O. CHUNG AND JIANG LUH
}

\begin{abstract}
The authors recently proved that for a semiprime ring without 2-torsion, a nilpotent derivation must have odd nilpotency. In this paper, we show the intriguing phenomenon that for a semiprime ring with characteristic 2 , the nilpotency of a nilpotent derivation must be of the form $2^{n}$. Combining these two results, we show that for a general semiprime ring with no torsion condition, the nilpotency of a nilpotent derivation is either odd or a power of 2 .
\end{abstract}

Let $R$ be a semiprime ring and $\partial$ a derivation of $R$. $\partial$ is said to be nilpotent if $\partial^{m} R=(0)$ for some positive integer $m$. The smallest such $m$ is called the nilpotency of $\partial$. It was proved in [1] that the nilpotency of $\partial$ is always odd if $R$ is 2-torsion free. The purpose of this note is to settle the general case without any torsion condition on $R$. It is known that the nilpotency of $\partial$ is either a power of 2 or an odd number. This result will be used in [2] to show the invariance of the nilpotency of a derivation on any nonzero ideals.

Let us first consider the complementary case that $R$ is of characteristic 2 .

THEOREM 1. Let $R$ be a semiprime ring of characteristic 2. Suppose $\partial$ is a derivation of $R$ and $\partial^{m}=0$ where $2^{k}<m \leq 2^{k+1}-1$ for some positive integer $k$. Then $\partial^{2^{k}}=0$ and hence the nilpotency of $\partial$ is a power of 2 .

PROOF. We proceed by induction on $k$. If $k=1$, then $m=3$ and $\partial^{3}=0$. For any $x, y \in R, 0=\partial^{3}(\partial x y)=\partial^{2} x \partial^{2} y$. By replacing $y$ by $y x$ and noting that $\partial^{2}$ is also a derivation of $R$, we obtain $0=\partial^{2} x \partial^{2}(y x)=\partial^{2} x\left(\partial^{2} y x+y \partial^{2} x\right)=\partial^{2} x y \partial^{2} x$ and hence by the semiprimeness of $R, \partial^{2} x=0$ for all $x \in R$.

We now assume $k>1$.

Case 1. Suppose $m<2^{k+1}-1$. Then $m=\sum_{j=0}^{k} \alpha_{j} 2^{j}$ where each $\alpha_{j}$ is either 0 or 1 and at least one of the $\alpha_{j}$ 's is zero. Let $i$ be the smallest one with $\alpha_{i}=0$. If $i=0$, then $m=2 m_{0}$ where $m_{0}=\sum_{j=1}^{k} \alpha_{j} 2^{j-1}$ and $2^{k-1}<m_{0} \leq 2^{k}-1$. Set $\delta=$ $\partial^{2}$. Then $\delta$ is a derivation with $\delta^{m_{0}}=0$. By the induction hypothesis, $\delta^{2^{k-1}}=0$ or $\partial^{2^{k}}=0$. Now assume $i>0$. Then $\alpha_{0}=1$ and $m+1=2^{i}+\sum_{j=i+1}^{k} \alpha_{j} 2^{j}=2^{i} n$ where $n=1+\sum_{j=i+1}^{k} \alpha_{j} 2^{j-i}$. Let $\delta=\partial^{2^{i}}$. Then $\delta$ is a derivation of $R$ and $2^{k-i}<n \leq 2^{k-i+1}-1$. Again by induction hypothesis, $\delta^{2^{k-i}}=0$ or $\partial^{2^{k}}=0$.

Case 2. Suppose $m=2^{k+1}-1$. In view of Case 1 , we need only show that $\partial^{m-1}=0$. Suppose to the contrary that $\partial^{m-1} \neq 0$. Then since, for any $x, y \in R$, $0=\partial^{m}\left(\partial^{m-2} x y\right)=\partial^{m-1} x \partial^{m-1} y$, there exists $a \in \partial^{m-1} R, a \neq 0$, such that $\partial a=0$ and $\partial^{m-1} x a=a \partial^{m-1} y=0$ for all $x, y \in R$. Let $I$ be the ideal of $R$ generated by $\partial^{m-1} R$ and let $S=\{(s, t) \mid s$ and $t$ are positive integers such that

Received by the editors September 22, 1983.

1980 Mathematics Subject Classification. Primary 16A72; Secondary 16A12.

Key words and phrases. Derivations, semiprime rings, nilpotency.

(c) 1984 American Mathematical Society $0002-9939 / 84 \$ 1.00+\$ .25$ per page 
$\exists b \in I$ with $\left.b \neq 0, \partial b=0, \partial^{s} R b=b \partial^{t} R=(0)\right\}$. Clearly $(m-1, m-1) \in S$. Partially order $S$ the following way: $\left(s_{1}, t_{1}\right) \prec\left(s_{2}, t_{2}\right)$ iff $s_{1} \leq s_{2}$ and $t_{1} \leq t_{2}$. let $(p, q)$ be a minimal element in $S$ and $c$ be a nonzero element in $I$ such that $\partial c=0$ and $\partial^{p} R c=c \partial^{q} R=(0)$.

If one of $p$ and $q$ is $\leq 2^{k}$, say $q \leq 2^{k}$, then $0=c \partial^{2^{k}}(x y)=c x \partial^{2^{k}} y$ for all $x, y \in R$, and consequently $c I=(0)$. Thus, by the semiprimeness of $R, c=0$, a contradiction. Hence both $p$ and $q$ are greater than $2^{k}$. For any $x, y \in R$,

$$
\begin{aligned}
0 & =\partial^{m}\left(\partial^{p-2^{k}-1} x c \partial^{q-2^{k}} y\right)=\partial^{2^{k}-1} \partial^{2^{k}}\left(\partial^{p-2^{k}-1} x c \partial^{q-2^{k}} y\right) \\
& =\partial^{2^{k}-1}\left(\partial^{p-1} x c \partial^{q-2^{k}} y\right)=\partial^{p-1} x c \partial^{q-1} y .
\end{aligned}
$$

If $c \partial^{q-1} y=0$ for all $y \in R$, then $(p, q-1) \in S$, contradicting the minimality of $(p, q)$ in $S$. Hence $c_{0}=c \partial^{q-1} y_{0} \neq 0$ for some $y_{0} \in R$. Evidently, $c_{0} \in I$, $\partial c_{0}=0$ and moreover, $c_{0} \partial^{q} y=c \partial^{q-1} y_{0} \partial^{q} y=c \partial^{2}\left(\partial^{q-1} y_{0} y\right)=0$ for all $y \in R$. Thus $(p-1, q) \in S$, again contradicting the minimality of $(p, q)$ in $S$. Therefore $\partial^{m-1}=0$.

Now we are in a position to prove our second result.

THEOREM 2. Let $R$ be a semiprime ring and $\partial$ a nilpotent derivation of $R$. Then the nilpotency of $\partial$ is either a power of 2 or an odd number.

Proof. Let $I=\{x \in R \mid 2 x=0\}$. The theorem follows immediately from [1] if $I=(0)$. We thus assume that $I \neq(0)$. Note that $R / I$ is a 2 -torsion free semiprime ring and the induced derivation $\bar{\partial}$ of $R / I$ defined by $\bar{\partial}(x+I)=\partial x+I$ is nilpotent. According to $[\mathbf{1}]$ the nilpotency of $\bar{\partial}$ is an odd number, say $2 n+1$, i.e., $\partial^{2 n+1} R \subseteq I$ but $\partial^{2 n} R \nsubseteq I$. On the other hand, $I$. itself is a semiprime ring of characteristic 2 and the restriction of $\partial$ on $I$ is a nilpotent derivation of $I$. By Theorem 1 the nilpotency of $\partial$ on $I$ is a power of 2 , say $2^{k}$. We claim that $\partial^{2^{k}} R \cap I=(0)$. Indeed, for any $a \in I$ and $x \in R, 0=\partial^{2^{k}}(x a)=\partial^{2^{k}} x a+x \partial^{2^{k}} a=\partial^{2^{k}} x a$ by noting that $2 a=0$, i.e. $\partial^{2^{k}} R \subseteq I^{l}$, the left annihilator of $I$. Consequently, $\partial^{2^{k}} R \cap I \subseteq I^{l} \cap I$ which is (0) by the semiprimeness of $R$. Thus $\partial^{2^{k}} R \cap I=(0)$.

If $2 n+1>2^{k}$, then $\partial^{2 n+1} R \subseteq \partial^{2^{k}} R \cap I$ and hence $\partial^{2 n+1} R=(0)$. The nilpotency of $\partial$ is $2 n+1$ as $\partial^{2 n} R \neq(0)$.

If $2 n+1<2^{k}$, then $\partial^{2^{k}} R \subseteq \partial^{2 n+1} R \subseteq I$. It follows that $\partial^{2^{k}} R=(0)$ and since (0) $\neq \partial^{2^{k}-1} I \subseteq \partial^{2^{k}-1} R$, the nilpotency of $\partial$ is $2^{k}$. This completes the proof.

We should note that in Theorems 1 and 2, the hypothesis that $R$ is semiprime is essential. It can be seen from the following example.

EXAMPLE. Let $R$ be the ring of $6 \times 6$ upper triangular matrices over $G F(2)$ and $\partial$ the inner derivation determined by the matrix $A=E_{12}+E_{23}+E_{34}+E_{45}+E_{56}$, where $E_{i j}$ denotes the unit matrix having 1 at the $(i, j)$-position and zero elsewhere. One can see easily that $A^{6}=0, A^{5} \neq 0$ and the nilpotency of $\partial$ is 6 which is neither a power of 2 not an odd number.

\section{REFERENCES}

1. L. O. Chung and J. Luh, Nilpotency of derivations, Canad. Math. Bull. 26 (1983), 34-346.

2. __ Nilpotency of derviations on an ideal, Proc. Amer. Math. Soc. 90 (1984), 211-214.

Department of Mathematics, North Carolina State University, Raleigh, NORTH CAROLINA 27695-8205 